\title{
Crystal structure of laser-induced subsurface modifications in $\mathrm{Si}$
}

\author{
P. C. Verburg ${ }^{1}$ L. A. Smillie ${ }^{2}$ G. R. B. E. Römer ${ }^{1}$ B. Haberl ${ }^{2,3}$. \\ J. E. Bradby ${ }^{2}$ J. S. Williams ${ }^{2}$ - A. J. Huis in 't Veld ${ }^{1}$
}

Received: 8 March 2015 / Accepted: 13 May 2015/Published online: 4 June 2015

(c) The Author(s) 2015. This article is published with open access at Springerlink.com

\begin{abstract}
Laser-induced subsurface modification of dielectric materials is a well-known technology. Applications include the production of optical components and selective etching. In addition to dielectric materials, the subsurface modification technology can be applied to silicon, by employing near to midinfrared radiation. An application of subsurface modifications in silicon is laser-induced subsurface separation, which is a method to separate wafers into individual dies. Other applications for which proofs of concept exist are the formation of waveguides and resistivity tuning. However, limited knowledge is available about the crystal structure of subsurface modifications in silicon. In this work, we investigate the geometry and crystal structure of laser-induced subsurface modifications in monocrystalline silicon wafers. In addition to the generation of lattice defects, we found that transformations to amorphous silicon and $\mathrm{Si}$-III/Si-XII occur as a result of the laser irradiation.
\end{abstract}

\section{Introduction}

Laser-induced subsurface modifications inside dielectric materials have been studied for a broad variety of applications [1]. The subsurface modification technology is

P. C. Verburg

p.c.verburg@utwente.nl

$\triangle$ G. R. B. E. Römer

g.r.b.e.romer@utwente.nl

1 Chair of Applied Laser Technology, Faculty of Engineering Technology, University of Twente, P.O. Box 217, 7500 AE Enschede, The Netherlands

2 Research School of Physics and Engineering, The Australian National University, Acton 2601, ACT, Australia

3 Present Address: Chemical and Engineering Materials Division, Oak Ridge National Laboratory, Oak Ridge, TN 37831, USA based on tightly focussing short lasers pulses inside a material, thereby creating localized changes to its crystal structure. Engineering applications of laser-induced subsurface modifications include the production of optical components [2-6], three-dimensional data storage [7], selective etching [8-10], selective metallization [11], twophoton polymerization [12] and improving the strength of glass [13]. Moreover, there is an interest in subsurface modifications for the study of matter under conditions of high pressure, since pressures in the TPa range can be induced when focussing laser pulses inside bulk materials [14-17].

This work is focussed on the formation of subsurface modifications in silicon. As silicon is the material of choice for the semiconductor industry, it is of interest to further develop the subsurface modification technology for this material. One of the most successful applications of subsurface modifications in silicon is dry and nearly debrisfree wafer dicing $[18,19]$, i.e. the separation of silicon wafers into individual dies. Moreover, the formation of optical components in silicon is of interest, as these components can be combined with integrated circuits on a single chip [20]. Finally, laser-induced subsurface modifications may be employed for resistivity tuning [21, 22].

The main requirement to produce subsurface modifications in any material is that the laser energy can be selectively absorbed in a confined volume. Various processing conditions have been investigated for the formation of modifications inside silicon. Nanosecond pulses with a wavelength $\lambda$ of $1.06 \mu \mathrm{m}$, which corresponds to a photon energy near the band gap, were found to result in modifications that coincide with the focus of the laser beam, without inducing surface damage [18, 23]. The same holds for $\lambda=1.55 \mu \mathrm{m}$ combined with short nanosecond pulses $[24,25]$. 
In addition to nanosecond pulses, femtosecond pulses with different wavelengths have been tested. Several femtosecond studies were based on line scans. These resulted in a combination of surface damage and modifications at a shallow depth $(\lambda=0.8 \mu \mathrm{m})$ [26, 27], modifications just below an oxide overcoat $(\lambda=2.4 \mu \mathrm{m})$ [20], a combination of surface damage and subsurface modifications at an unknown depth $(\lambda=1.55 \mu \mathrm{m})[28]$ and modifications near the back surface of a substrate $(\lambda=1.55 \mu \mathrm{m})$ [29]. In other femtosecond studies, single or multiple laser pulses were applied to a single location. During these experiments, using laser pulses with wavelengths of $1.2 \mu \mathrm{m}$ [30] and $1.3 \mu \mathrm{m}$ [31], no subsurface damage was found, even when irradiating the same location with a large number of pulses. The same result was obtained for picosecond pulses $(\lambda=1.03 \mu \mathrm{m})$ [32].

Based on the experiments discussed above, the parameter window for the formation of subsurface modifications can be established. However, limited information is available about the crystal structure of modifications inside silicon after laser processing. This information is required to assess the feasibility of specific applications of subsurface modifications and to understand their operating principles. Suggestions of changes to the material structure include the formation of polycrystalline material and voids [18] and the formation of a high density of dislocations [33]. Moreover, molecular dynamics simulations predicted the production of amorphous silicon [34]. Finally, Raman spectroscopy measurements have shown that transformations to amorphous and polycrystalline silicon occur [29].

The objective of this work is to investigate the geometry and crystal structure of laser-induced subsurface modifications in crystalline silicon wafers, based on an experimental approach.

\section{Laser processing and sample analysis}

To establish the geometry and crystal structure of subsurface modifications, a number of steps are required. First, the modifications must be produced by tightly focussing laser pulses inside a silicon wafer. Next, unless a nondestructive analysis method suffices, the modifications have to be exposed on a surface or in a lamella. Finally, the material structure of the modifications can be analysed. In this section, an overview is given of the experimental methods to accomplish these steps.

\subsection{Laser processing conditions}

Two processing strategies have been used to fabricate subsurface modifications inside silicon. These strategies are based on different laser wavelengths, as listed below.
1. A wavelength of $1061 \mathrm{~nm}$, corresponding to a photon energy near the band gap of silicon. At this wavelength, the linear interband absorption coefficient strongly depends on temperature [35]. Consequently, a thermal runaway can be triggered $[18,36]$.

2. A wavelength of $1549 \mathrm{~nm}$, which corresponds to a photon energy well below the band gap. This strategy depends on two-photon absorption to initiate the absorption of laser energy.

Based on numerical simulations and experiments, the required energy to modify bulk silicon was found to be consistent with the energy needed to melt silicon [24, 36]. Therefore, the hypothesis regarding the material modification is that melting and fast resolidification is the primary damage mechanism. This implies that an energy density of $7.1 \cdot 10^{3} \mathrm{~J} / \mathrm{cm}^{3}$ is required to induce bulk modifications [24]. Depending on the volume in which the laser energy is deposited, this energy density determines the laser pulse energy that should be selected.

Apart from the wavelength and pulse energy, a suitable pulse duration has to be chosen. Pulses that are too long result in excessive conduction of heat and free carriers during the pulse. Moreover, for the 1549-nm process, a pulse that is too long in duration yields insufficiently high laser intensities in the focus to enable effective two-photon absorption. However, for both wavelengths, a lower boundary to the pulse duration also exists. For a fixed pulse energy, shorter pulses result in higher instantaneous powers of the laser beam. Excessive powers induce two-photon absorption above the focus [36, 37], resulting in absorption of laser energy at undesired locations and plasma-induced defocussing of the laser beam [37]. Moreover, when a critical power threshold is exceeded, self-focussing due to the Kerr effect becomes an issue [16]. The issues concerning the use of excessive instantaneous power levels explain the limited success of femtosecond experiments (see Sect. 1).

Detailed information about the set-up that was used to tightly focus laser pulses inside crystalline silicon can be found in Ref. [24]. The focussing objective that was employed is manufactured by Leica Microsystems and has type number 11101666 . It has a numerical aperture of 0.7 and a coverslip correction for $100 \mu \mathrm{m} \mathrm{Si}$. When creating samples that were subject to destructive analysis techniques, modifications had to be created close to the sample surface. Therefore, for these samples, a $250 \mu \mathrm{m}$-thick quartz window was used to protect the objective, resulting in negative spherical aberrations.

Two laser sources were attached to the set-up. The first is an MWTechnologies MOPA-LF-1550 erbium-doped fibre laser emitting at $1549 \mathrm{~nm}$. It has a fixed full width at half maximum pulse duration of $3.5 \mathrm{~ns}$. The second is an 
SPI SP-020P-A-EP-S-A-Y ytterbium-doped fibre laser with a wavelength of $1061 \mathrm{~nm}$. This laser source has a tunable pulse duration. Since the $1061 \mathrm{~nm}$ process supports a wide range of pulse durations [36], the ability to tune the pulse shape enables the influence of the pulse duration to be investigated. The range of pulse durations that the SPI source can generate was measured by a fast photodiode ( $2 \mathrm{GHz}$ bandwidth) and was found to be 2-460 ns.

The estimated spot sizes inside silicon $\left(1 / e^{2}\right.$ diameter), when the focus depth is matched to the coverslip correction, are $2.37 \mu \mathrm{m}$ for the $1549-\mathrm{nm}$ wavelength and $1.92 \mu \mathrm{m}$ for the 1061-nm wavelength. The beam qualities of the laser sources and the diameters of the laser beams at the back aperture of the objective have been taken into account in these estimates. The laser pulse energies were measured at the location of the sample.

\subsection{Sample preparation and analysis}

Four different techniques were used to analyse the geometry and crystal structure of the laser-induced subsurface modifications. These are infrared microscopy, scanning electron microscopy (SEM), transmission electron microscopy (TEM) and Raman spectroscopy.

Infrared microscopy is a non-destructive technique that requires no further sample preparation after laser processing. For infrared microscopy, an optical microscope equipped with a transmitted near-infrared light source was employed. A regular silicon CMOS camera was used to observe the infrared light. Advantages of infrared microscopy are that the modifications can easily be located and that any features that are observed are guaranteed to be a direct result of the laser-material interaction. A disadvantage is its limited resolution.

For SEM, samples were fractured along subsurface modifications to expose them on a surface. The destructive sample preparation adds some uncertainty regarding the cause of the observed features. The fracturing may result in additional damage beyond the damage induced by the laser-material interaction. Moreover, part of the damage could remain hidden.

The Raman spectroscopy system that was employed is equipped with a HeNe laser source emitting at $633 \mathrm{~nm}$. The spot size on the sample was approximately $2 \mu \mathrm{m}$.

Rather than fracturing, samples intended for Raman spectroscopy, or the preparation of TEM lamellae perpendicular to the optical axis of the laser beam (see Fig. 1), were mechanically polished. The main advantage of polishing over fracturing is the lower roughness of the exposed surface. First, parallel sets of subsurface modifications were created, which were approximately aligned with a $\{110\}$ plane, one intended for analysis, while the other was fractured. This fractured plane was used as the starting plane for polishing.
For the fabrication of TEM lamellae, electron-transparent lamellae were produced by focussed ion beam (FIB) milling. For the TEM lamellae, the laser modifications were spaced $20 \mu \mathrm{m}$ apart to prevent any interaction between neighbouring pulses. For Raman spectroscopy, a spacing between the modifications of $2 \mu \mathrm{m}$ was employed to sample a large number of modifications. However, this does imply that the crystal structure of laser-modified material may undergo additional changes due to subsequent irradiation by neighbouring pulses.

To obtain TEM lamellae containing cross sections of modifications along the optical axis (see Fig. 1), modifications were created close to the wafer surface, while producing surface grooves to mark their positions. Next, lamellae were directly created by FIB milling and then "plucked out" in situ.

\section{Results}

The wafers that were selected for the experiments are $160-\mu \mathrm{m}$ thick monocrystalline silicon wafers with a $<100>$ crystal orientation and a moderate boron doping. The resistivity of the wafers was measured as $10.3 \Omega \mathrm{cm}$. The corresponding density of free carriers is sufficiently low to prevent appreciable free carrier absorption by the intrinsic concentration of free carriers [38].

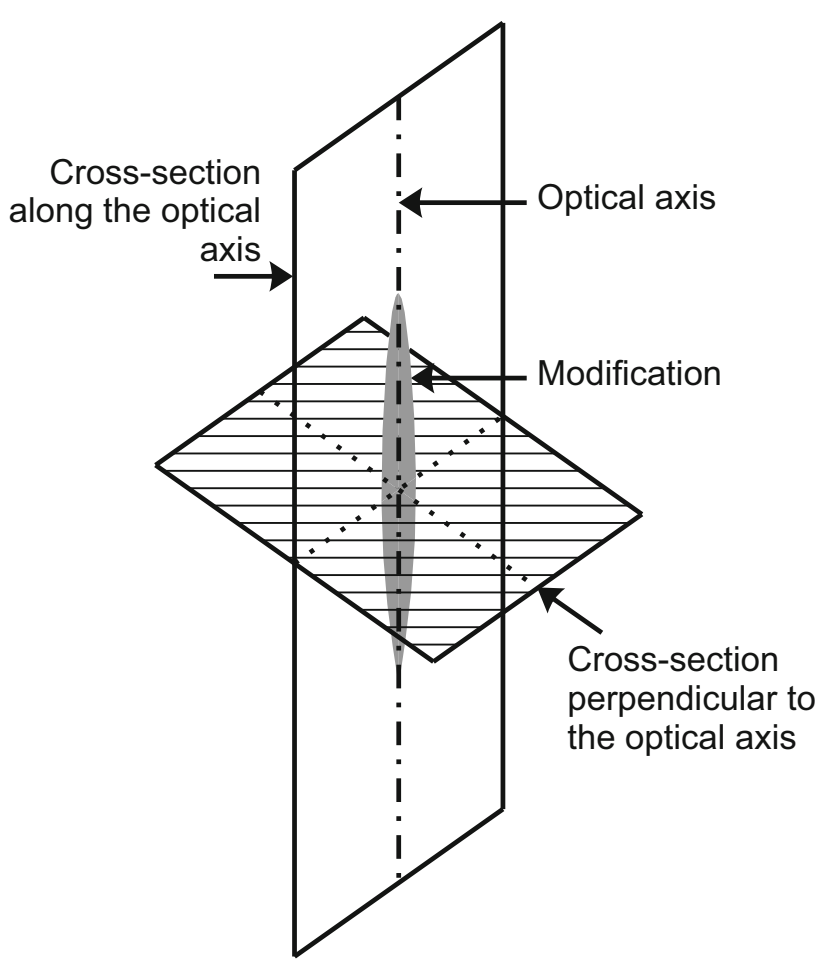

Fig. 1 Drawing of the orientations of the TEM lamellae with respect to the modifications and the optical axis 
In this section, the results obtained using the analysis techniques discussed in Sect. 2.2 are presented. The interpretation of these results in terms of the modification mechanisms that occurred during the laser-material interaction can be found in Sect. 4 .

\subsection{Infrared microscopy}

First, infrared microscopy was employed to establish the parameter window for the formation of subsurface modifications. Subsurface modifications were successfully created using all combinations of wavelengths and pulse durations that can be generated by the laser sources described in Sect. 2.1. However, the range of suitable pulse energies was found to be narrow for a 2-ns pulse duration combined with a wavelength of $1061 \mathrm{~nm}$. Modifications were only detected for pulse energies between 0.14 and $0.91 \mu \mathrm{J}$, while changing the pulse energy in steps of $0.07 \mu \mathrm{J}$. Their visibility in infrared microscopy was poor at pulse energies of $0.7 \mu \mathrm{J}$ and above. This implies that the pulse duration of $2 \mathrm{~ns}$ is close to the boundary of the process window, which is consistent with numerical simulations [36]. For 1061-nm pulses with a duration of $8 \mathrm{~ns}$, clearly visible modifications were found for all pulse energies above the modification threshold of $\approx 0.2 \mu \mathrm{J}$ that were investigated (up to $4 \mu \mathrm{J}$ ). Previous experiments demonstrated that for the $1549 \mathrm{~nm} / 3.5 \mathrm{~ns}$ process, the size of the subsurface modifications may be tuned by varying the pulse energy between 0.4 and $4 \mu \mathrm{J}$ [24].

In spite of the limited resolution of infrared microscopy, clear differences in the geometry of the modifications were found, when comparing short and long nanosecond pulses. A comparison between 8- and 460-ns pulses with a wavelength of $1061 \mathrm{~nm}$, while keeping the pulse energy constant, is shown in Fig. 2. While no cracks are visible for the 8-ns pulse duration, the formation of cracks that sometimes interconnect the modifications can be observed for the 460-ns pulses. For 3.5-ns pulses with a wavelength of $1549 \mathrm{~nm}$, cracks perpendicular to the optical axis were not visible by infrared microscopy.

\subsection{Scanning electron microscopy}

Figure 3 shows an overview of the side wall of a die that was separated by fracturing it along laser-induced subsurface modifications, created by laser pulses with a $1549-\mathrm{nm}$ wavelength. Vertical lines that coincide with the transverse spacing between the laser pulses can be observed. The laser modifications become more chaotic further away from the focus of the laser beam. This may be related to accumulated damage from multiple laser pulses. The Gaussian beam diameter in focus is comparable with the spacing

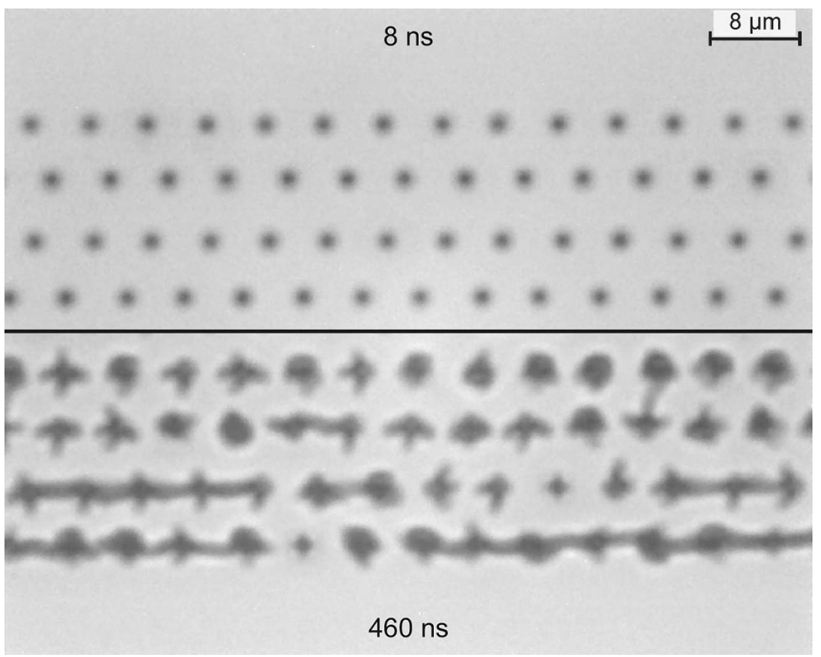

Fig. 2 Infrared transmission micrograph of subsurface modifications inside silicon (top view). Eight horizontal laser tracks were produced containing modifications spaced $5 \mu \mathrm{m}$ apart. Pulse energy: $2.8 \mu \mathrm{J}$, wavelength: $1061 \mathrm{~nm}$, focal spot: $100 \mu \mathrm{m}$ below the surface. Pulse duration: $8 \mathrm{~ns}$ (top), $460 \mathrm{~ns}$ (bottom)

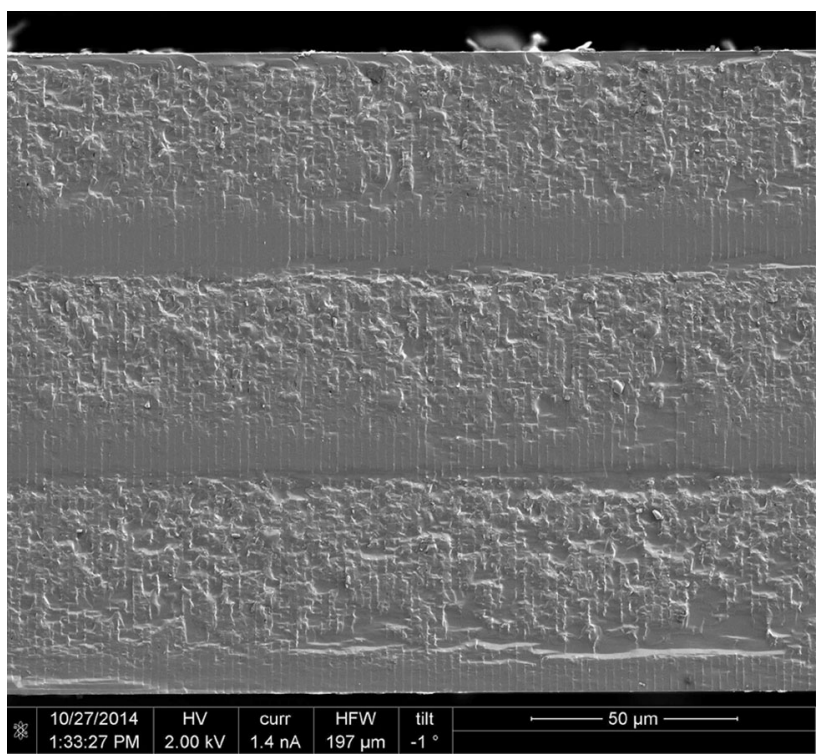

Fig. 3 SEM micrograph (secondary electrons) of a fracture plane obtained after dicing a $160 \mu \mathrm{m}$-thick silicon wafer. Wavelength: $1549 \mathrm{~nm}$, pulse duration: $3.5 \mathrm{~ns}$, pulse energy: $2 \mu \mathrm{J}$, transverse spacing modifications: $2 \mu \mathrm{m}$. Three different focus depths inside the wafer were employed. The laser beam propagation direction is from top to bottom

between the modifications. Hence, a significant overlap between laser spots is present away from the focal plane.

Moreover, randomly distributed voids were found. A detail of a number of voids is shown in Fig. 4. Perpendicular to the optical axis, the dimensions of the voids are in the order of $100-500 \mathrm{~nm}$. Matching voids were 


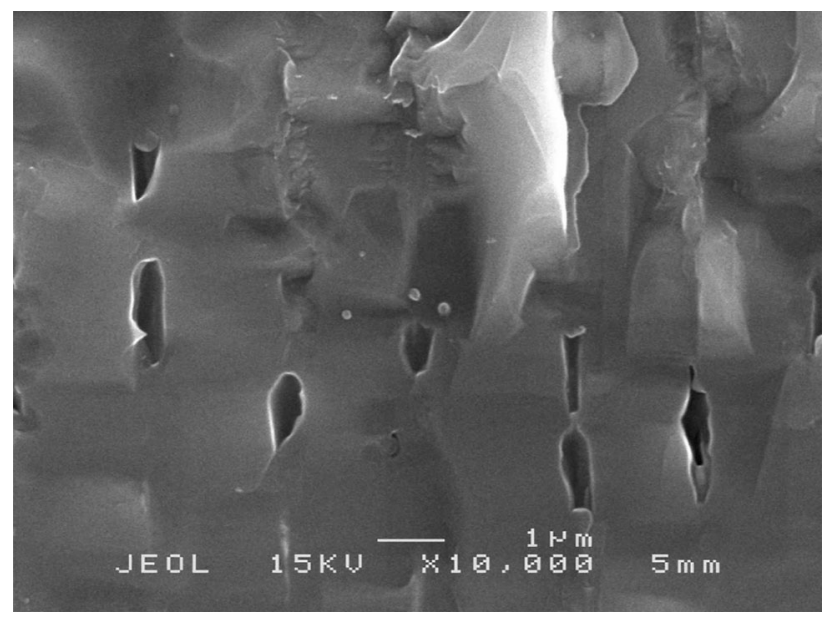

Fig. 4 SEM micrograph (secondary electrons) of a detail of a fracture plane obtained after dicing a $160 \mu \mathrm{m}$-thick silicon wafer. Wavelength: $1549 \mathrm{~nm}$, pulse duration: $3.5 \mathrm{~ns}$, pulse energy: $1.3 \mu \mathrm{J}$, transverse spacing modifications: $2 \mu \mathrm{m}$. The laser beam propagation direction is from top to bottom

generally found on both sides of the fracture plane. This suggests that the voids were already present before fracturing, although the possibility that nanometre-scale particles escaped from the sample during fracturing cannot be ruled out.

The formation of voids as a result of focussing laser pulses inside bulk materials might be an indicator of the presence of high-pressure silicon phases that are more dense than diamond cubic silicon, since one mechanism to produce such voids is by confined microexplosions that generate pressures in the GPa to TPa range [14-17]. Regardless of the mechanism for forming voids, the presence of voids in the bulk implies, by mass conservation, the presence of corresponding regions of higher density than unstrained diamond cubic silicon. Therefore, establishing whether high-pressure silicon phases are present was a specific goal of the analysis of subsurface modifications by TEM and Raman spectroscopy (see Sect. 3.3 and 3.4).

\subsection{Transmission electron microscopy}

A scanning transmission electron microscopy (STEM) high-angle annular darkfield (HAADF) micrograph of a cross section along the optical axis of the laser beam is shown in Fig. 5. Two modifications that were created by a single laser pulse are visible. STEM was selected instead of conventional TEM, as the lamella had to be kept relatively thick to maintain its structural integrity.

The irregular cracks that run from the top of the modifications to the protective platinum layer on the wafer surface appeared during the ion beam milling and are not a

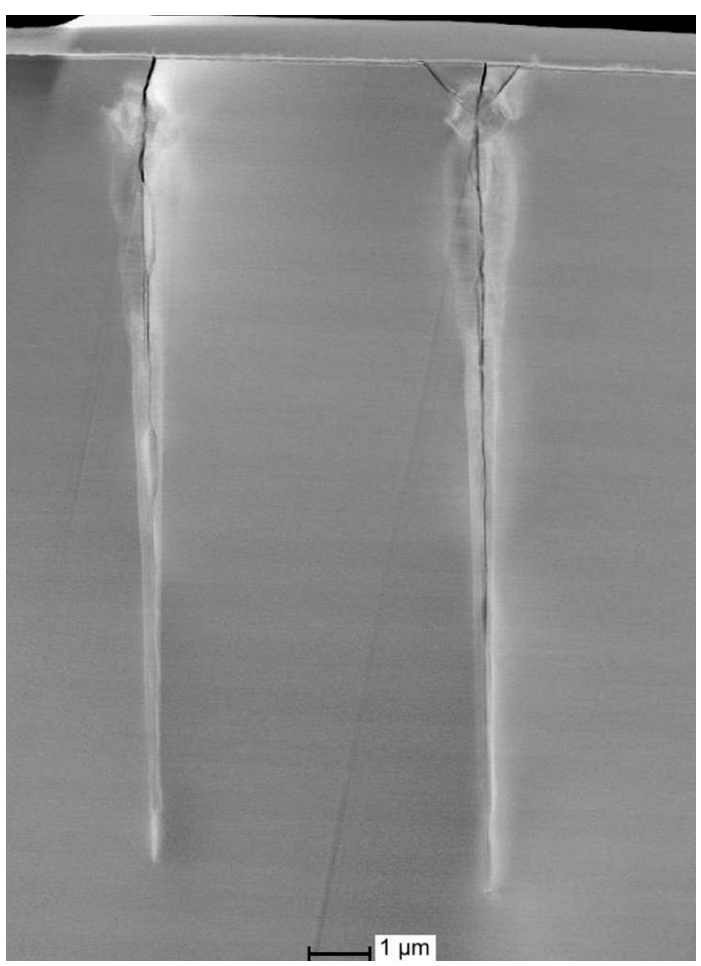

Fig. 5 STEM-HAADF micrograph of two subsurface modifications obtained by stitching two images (top and bottom). Cross section: along the optical axis. Transverse spacing modifications: $5 \mu \mathrm{m}$. Wavelength: $1549 \mathrm{~nm}$, pulse duration: $3.5 \mathrm{~ns}$, pulse energy: $0.6 \mu \mathrm{J}$. The propagation direction of the laser beam is from top to bottom. A platinum layer was deposited on the wafer surface before ion milling

direct result of the laser process. Cracks are also visible inside the modifications, which coincide with the optical axis of the beam. Since these cracks were not present in lamellae perpendicular to the optical axis, they should also be attributed to stress relief during the ion milling process.

A conventional TEM micrograph of a cross section perpendicular to the optical axis of the laser beam is shown in Fig. 6. This cross section corresponds to a location within a few micrometres of the focus of the laser beam. In Fig. 6, the followings features can be observed: a square pattern centred on the optical axis; defect lines radiating out from the corners of the square pattern; unmodified material surrounding the square pattern with the same diamond cubic crystal structure and orientation as the unprocessed wafer; and bend contours within the TEM lamella.

Additional TEM micrographs of cross sections perpendicular to the optical axis of the laser beam are shown in Figs. 7 and 8. These cross sections also correspond to a location close to the focal plane. Moreover, the modifications were produced using the same wavelength, pulse energy and pulse duration. Nevertheless, significant stochastic variations are present. Based on selected area 


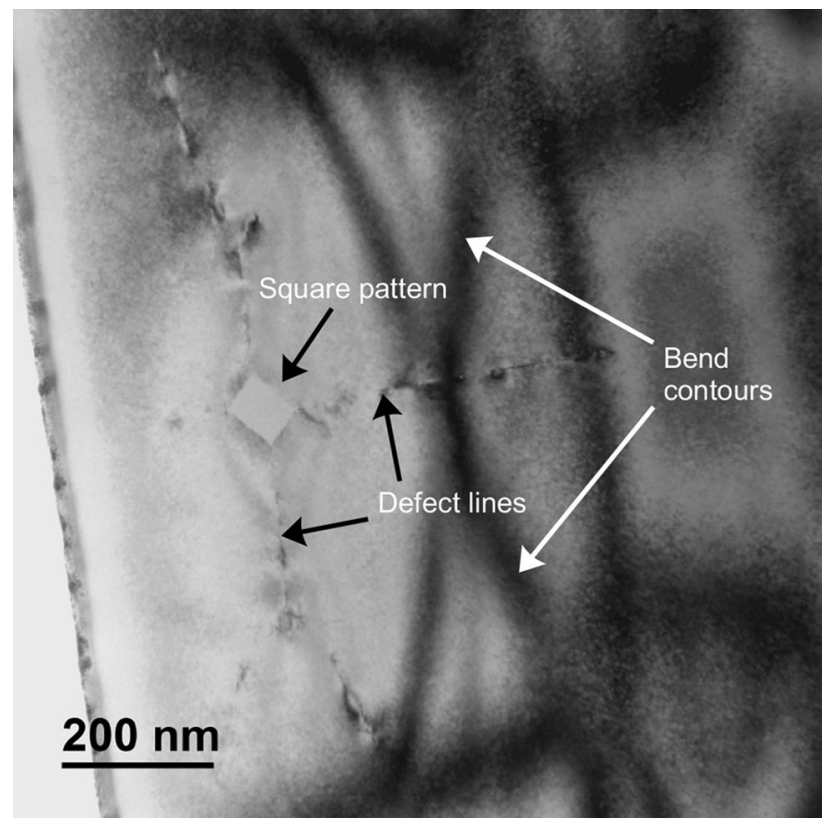

Fig. 6 TEM micrograph (brightfield) of a subsurface modification. Cross section: perpendicular to the optical axis. Wavelength: $1549 \mathrm{~nm}$, pulse duration: $3.5 \mathrm{~ns}$, pulse energy: $2 \mu \mathrm{J}$

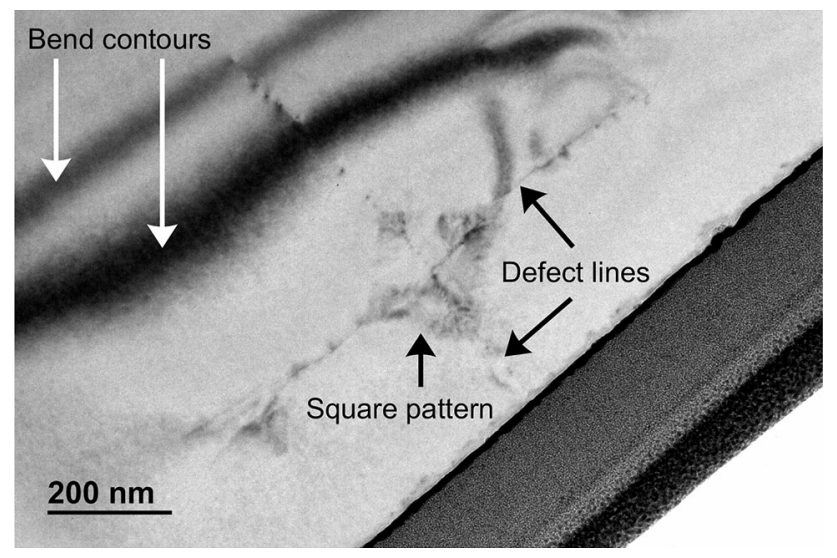

Fig. 7 TEM micrograph (brightfield) of a subsurface modification. Cross section: perpendicular to the optical axis. The processing conditions used to generate this modification were identical to those used for Figs. 6 and 8

diffraction patterns that were recorded, it was found that the square pattern in Fig. 6 is amorphous, while diamond cubic silicon with a high concentration of lattice defects is present near the optical axis in Figs. 7 and 8. The crystalline material with defects has the same orientation as the base material. No holes are visible to associate with the voids shown in Fig. 4. This may be due to the fact that the chance of finding a void in TEM cross sections is small because of their random distribution and low density.

In two modifications, out of a total of 27 that were analysed, crystalline material was detected at a location along one of the defect lines that was not diamond cubic

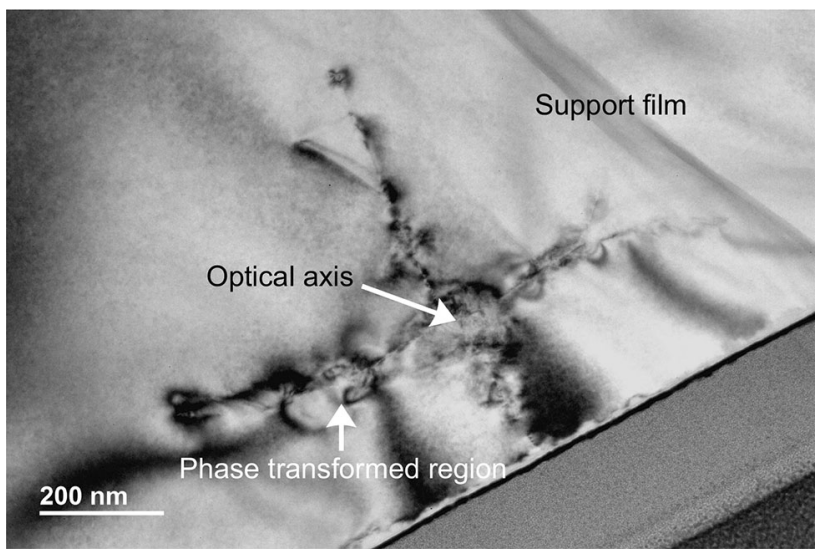

Fig. 8 TEM micrograph (brightfield) of a subsurface modification. Cross section: perpendicular to the optical axis. The processing conditions used to generate this modification were identical to those used for Figs. 6 and 7

silicon. Indeed, there were diffraction spots in selected area diffraction patterns that did not match the $d$-spacing of any set of planes corresponding to diamond cubic silicon. This was the case for the modification that is shown in Fig. 8 for the area labelled "phase-transformed region". The $d$-spacings of the additional spots matched $\mathrm{Si}$-III and $\mathrm{Si}$-XII that have previously been identified in nanoindentation studies [39] and fs laser irradiations [40] of silicon. The number of detectable diffraction spots from such phases was insufficient to identify the reciprocal lattice. Therefore, Raman spectroscopy was employed as a second means of obtaining information about the lattice structure of high-pressure phases within laser modifications (see Sect. 3.4).

\subsection{Raman spectroscopy}

In addition to selected area diffraction, Raman spectroscopy was employed to identify the phases that were present in the laser-modified material. A reference spectrum was recorded of material that was not modified by the laser beam. Any deviation from the reference spectrum indicates that laser-modified material may be present.

Phase-changed material was consistently found, with no clear correlation with the location along the optical axis. A comparison of the Raman spectra of unmodified and modified silicon is shown in Fig. 9. A strong peak is observed around a Raman shift of $360 \mathrm{~cm}^{-1}$, which indicates the presence of $\mathrm{Si}$-III/Si-XII phases [41]. Previously, both phases were found to coexist in nanoindentation experiments [41], and we suggest that this is also the case in the phase-transformed region of Fig. 8, although we have insufficient Raman and TEM diffraction data to confirm this. 
Fig. 9 Raman spectra from unmodified silicon and modified silicon on a fracture plane. The intensities were scaled such that the highest peak is equal to one. For clarity, one of the diamond cubic peaks at $521 \mathrm{~cm}^{-1}$ is not fully shown. Transverse spacing modifications: $2 \mu \mathrm{m}$.

Wavelength: $1549 \mathrm{~nm}$, pulse duration: $3.5 \mathrm{~ns}$, pulse energy: $2 \mu \mathrm{J}$

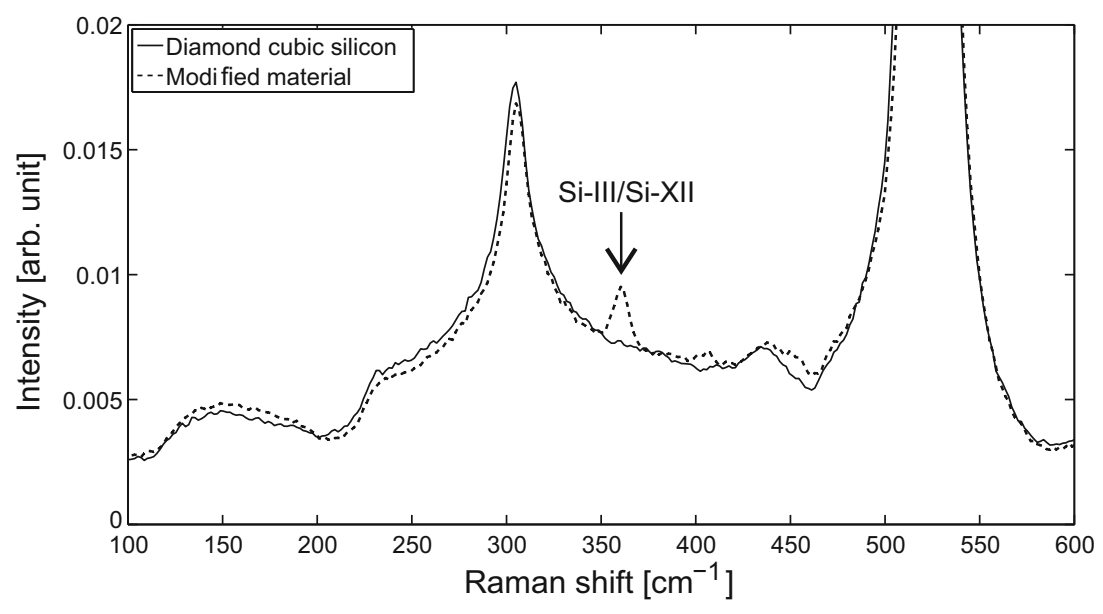

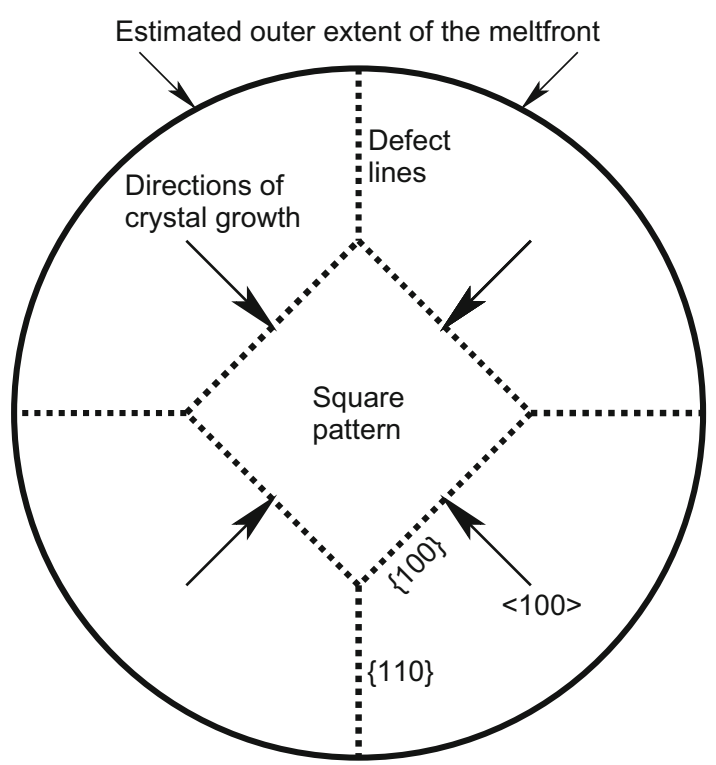

Fig. 10 Diagram of the shape of a cross section of a subsurface modification perpendicular to the optical axis

\section{Material modification mechanisms}

In this section, the material modification mechanisms that occurred during the laser-material interaction will be considered based on the results presented in Sect. 3.

\subsection{Melting and resolidification}

The brightfield TEM micrographs showed non-axisymmetric features including defect lines and a square pattern. A schematic diagram of the features observed on a cross section perpendicular to the optical axis is shown in Fig. 10. Note that the features that are indicated are the most common ones observed in TEM micrographs, although variations are present between different modifications.
The features in Fig. 10 can be explained based on the dynamics of solidification from a melt. The temperature profile radially out from the optical axis will determine the extent of the molten zone: its outer extremity will correspond to the distance where the temperature falls to the melting point of silicon. Resolidification will occur as the temperature rapidly drops via heat conduction to the surrounding solid. The melt-solid interface front can move inwards towards the optical axis at velocities of several $\mathrm{m} / \mathrm{s}$, with the front expected to speed up as it progresses, consistent with previous laser melting from the surface [42]. Epitaxial growth seeded by the unmodified diamond cubic silicon solid will occur, and this explains why most of the material outside of the square pattern is damage-free single crystal diamond cubic silicon. As a result of such perfect epitaxial silicon, it is not possible to determine the outer extent of the melt front and in Fig. 10 we provide a lower estimate of its penetration depth at the extremities of the defect lines. Indeed, the crystal directions of the defect lines and the $\{100\}$ facets of the square pattern suggest that the solidification (nucleation) of epitaxial silicon is favoured on such $\{100\}$ crystal facets. Hence, the defect lines would occur at the overlap of adjacent $\{100\}$ solidification fronts. The solid structure that is left behind after resolidification depends on the speed of the resolidification process. For crystal growth along a $<100>$ direction, crystalline silicon with defects or amorphous silicon is formed once the speed of the resolidification approaches or exceeds $15 \mathrm{~m} / \mathrm{s}$ [42]. Thus, the perfect epitaxial growth will cease along $\{100\}$ facets at such solidification velocities, leading to either defective crystalline material (Figs. 7 and 8) or amorphous silicon (Fig. 6) inside a square pattern as illustrated in Fig. 10. The dynamics of such solidification will be discussed in more detail in a forthcoming publication. 


\subsection{Pressure-induced phase changes and void formation}

Based on the selected area electron diffraction and Raman spectroscopy data, pressure-induced phase transformations were found to be a contributing damage mechanism. At least a pressure of approximately $10 \mathrm{GPa}$ is needed to phase transform diamond cubic silicon [39-41, 43]. Furthermore, if the voids are a direct result of the laser-material interaction, this implies that space has to be available for these voids to exist, due to either an increase in density elsewhere in the bulk of the material or deformation extending out to the outer surface of the sample, which is highly unlikely in silicon. $\mathrm{Si}-\mathrm{III} / \mathrm{Si}$-XII phases, which were detected in laser-processed samples, have a density of approximately $2.55 \cdot 10^{3} \mathrm{~kg} / \mathrm{m}^{3}$ at atmospheric pressure, compared with $2.34 \cdot 10^{3} \mathrm{~kg} / \mathrm{m}^{3}$ for diamond cubic silicon [44]. Consequently, it is plausible that the formation of voids and high-pressure phases is linked to conserve mass in the laser-modified region.

The formation of high pressures when tightly focussing laser pulses inside transparent materials is generally attributed to confined microexplosions [14-17], causing solid-solid phase transformations due to high pressures induced by a shockwave. However, the high-pressure phases that were detected during this work were found along defect lines that were presumably formed by the overlap of adjacent resolidification fronts, which suggests that a liquid-solid instead of solid-solid transition took place. According to the phase diagram of silicon [45], this is a realistic option, as the metallic $\mathrm{Si}$-II phase, which is $22 \%$ more dense than diamond cubic silicon, can be directly formed from a pressurized liquid. $\mathrm{Si}$-II will transform into $\mathrm{Si}$-III/Si-XII upon (partial) release of the pressure [39$41,43]$. We suggest that, at the overlap of $\{100\}$ solidification fronts, isolated melt volumes can be trapped in surrounding solid and the melt (which is $\approx 10 \%$ more dense than diamond cubic silicon) solidifies into $\mathrm{Si}-\mathrm{III} / \mathrm{Si}$-XII phases of a similar density.

\section{Conclusions and recommendations}

We analysed the geometry and crystal structure of laserinduced subsurface modifications in crystalline silicon. These modifications were created by tightly focussing nanosecond pulses with wavelengths of 1061 and $1549 \mathrm{~nm}$ inside silicon wafers.

The types of modifications of the bulk of monocrystalline diamond cubic silicon that we found are: (1) cracks and voids, (2) perfectly solidified diamond cubic silicon and silicon with lattice defects, (3) the formation of amorphous material and (4) the formation of $\mathrm{Si}-\mathrm{III} / \mathrm{Si}$-XII high-pressure phases.

While the laser beam was axisymmetric, the laser-induced subsurface modifications were not. This discrepancy is likely to be due to the regrowth of crystalline material from a melt, implying that melting and fast resolidification is the primary damage mechanism.

The high-pressure phases were detected in a position where the silicon has likely undergone melting and resolidification. Therefore, a different mechanism than a confined microexplosion during the laser pulse is expected to be responsible for their formation. Exploring the possibility to measure the presence of solid, liquid and vapour phases in real time during the laser pulse, using a pump-probe setup, is of interest for future research. Such information will help to clarify the formation mechanisms of the highpressure phases.

The exact extent of the molten volume could not be determined with certainty based on the transmission electron micrographs, since it is expected that the resolidification resulted in perfect epitaxial growth of diamond cubic silicon at the extremities of the molten zone. It is recommended to investigate options to resolve this issue during future research. A potential solution is to create laser modifications inside highly doped wafers, as the analysis of the transport of the dopant may enable the molten zone to be identified.

Acknowledgments The authors would like to thank Frank Rietveld, Harry Roberts and Xiao-Mei Zhang of NXP Semiconductors Regional Quality Center Nijmegen for the preparation of the STEM micrograph shown in Fig. 5, the Australian National Fabrication Facility for the use of their focussed ion beam and the Centre for Advanced Microscopy for the use of their transmission electron microscopes.

Open Access This article is distributed under the terms of the Creative Commons Attribution 4.0 International License (http:// creativecommons.org/licenses/by/4.0/), which permits unrestricted use, distribution, and reproduction in any medium, provided you give appropriate credit to the original author(s) and the source, provide a link to the Creative Commons license, and indicate if changes were made.

\section{References}

1. R.R. Gattass, E. Mazur, Nat. Photon. 2(4), 219 (2008)

2. K. Miura, J. Qiu, H. Inouye, T. Mitsuyu, K. Hirao, Appl. Phys. Lett. 71(23), 3329 (1997)

3. Y. Cheng, K. Sugioka, K. Midorikawa, M. Masuda, K. Toyoda, M. Kawachi, K. Shihoyama, Opt. Lett. 28(13), 1144 (2003)

4. Y. Cheng, H.L. Tsai, K. Sugioka, K. Midorikawa, Appl. Phys. A: Mater. Sci. Process. 85(1), 11 (2006)

5. H.B. Sun, Y. Xu, S. Juodkazis, K. Sun, M. Watanabe, S. Matsuo, H. Misawa, J. Nishii, Opt. Lett. 26(6), 325 (2001) 
6. Y. Hanada, K. Sugioka, I. Shihira-Ishikawa, H. Kawano, A. Miyawaki, K. Midorikawa, Lab Chip 11(12), 2109 (2011)

7. E. Glezer, M. Milosavljevic, L. Huang, R. Finlay, T.H. Her, J. Callan, E. Mazur, Opt. Lett. 21(24), 2023 (1996)

8. W.W. Hansen, S.W. Janson, H. Helvajian, Proc. SPIE 2991, 104 (1997)

9. S. Matsuo, S. Kiyama, Y. Shichijo, T. Tomita, S. Hashimoto, Y. Hosokawa, H. Masuhara, Appl. Phys. Lett. 93(5), 051107 (2008)

10. M. Masuda, K. Sugioka, Y. Cheng, T. Hongo, K. Shihoyama, H. Takai, I. Miyamoto, K. Midorikawa, Appl. Phys. A: Mater. Sci. Process. 78(7), 1029 (2004)

11. K. Sugioka, T. Hongo, H. Takai, K. Midorikawa, Appl. Phys. Lett. 86(17), 171910 (2005)

12. S. Maruo, O. Nakamura, S. Kawata, Opt. Lett. 22(2), 132 (1997)

13. M. Mirkhalaf, A.K. Dastjerdi, F. Barthelat, Nat. Commun. 5, 3166 (2014)

14. S. Juodkazis, K. Nishimura, S. Tanaka, H. Misawa, E.G. Gamaly, B. Luther-Davies, L. Hallo, P. Nicolai, V.T. Tikhonchuk, Phys. Rev. Lett. 96(16), 166101 (2006)

15. E.N. Glezer, E. Mazur, Appl. Phys. Lett. 71(7), 882 (1997)

16. E.G. Gamaly, S. Juodkazis, K. Nishimura, H. Misawa, B. LutherDavies, L. Hallo, P. Nicolai, V.T. Tikhonchuk, Phys. Rev. B 73(21), 214101 (2006)

17. L. Rapp, B. Haberl, J.E. Bradby, E.G. Gamaly, J.S. Williams, A.V. Rode, Appl. Phys. A: Mater. Sci. Process. 114(1), 33 (2014)

18. E. Ohmura, F. Fukuyo, K. Fukumitsu, H. Morita, J. Achiev. Mater. Manuf. Eng. 17, 381 (2006)

19. Y. Izawa, S. Tanaka, H. Kikuchi, Y. Tsurumi, N. Miyanaga, M. Esashi, M. Fujita, in IEEE 21st International Conference on Micro Electro Mechanical Systems (2008)

20. A.H. Nejadmalayeri, P.R. Herman, J. Burghoff, M. Will, S. Nolte, A. Tünnermann, Opt. Lett. 30(9), 964 (2005)

21. E. Boulais, J. Fantoni, A. Chateauneuf, Y. Savaria, M. Meunier, IEEE Trans. Electron Dev. 58(2), 572 (2011)

22. R. Singh, Y. Audet, Y. Gagnon, Y. Savaria, E. Boulais, M. Meunier, IEEE Trans. Briefs Circ. Syst. II, Exp 58(2), 75 (2011)

23. P.C. Verburg, G.R.B.E. Römer, A.J. Huis in 't Veld, in Proceedings of the 14th International Symposium on Laser Precision Microfabrication, Niigata, Japan 23-26 July 2013

24. P.C. Verburg, G.R.B.E. Römer, A.J. Huis in 't Veld, Opt. Express 22(18), 21958 (2014)

25. O. Tokel, A. Turnali, I. Pavlov, S. Tozburun, I. Akca, F.Ö. Ilday, arXiv:1409.2827 (2014)
26. C. Li, X. Shi, J. Si, F. Chen, T. Chen, Y. Zhang, X. Hou, Appl. Phys. B: Lasers Opt. 98(2-3), 377 (2010)

27. T. Chen, J. Si, X. Hou, S. Kanehira, K. Miura, K. Hirao, Appl. Phys. Lett. 93(5), 051112 (2008)

28. V.V. Parsi Sreenivas, M. Bülters, R.B. Bergmann, J. Europ. Opt. Soc. Rap. Public 7, 12035 (2012)

29. Y. Ito, H. Sakashita, R. Suzuki, M. Uewada, K.P. Luong, R. Tanabe, J. Laser Micro Nanoen. 9(2), 98 (2014)

30. V.V. Kononenko, V.V. Konov, E.M. Dianov, Opt. Lett. 37(16), $3369(2012)$

31. S. Leyder, D. Grojo, P. Delaporte, W. Marine, M. Sentis, O. Utéza, Proc. SPIE 8770, 877004 (2013)

32. P.C. Verburg, G.R.B.E. Römer, G.H.M. Knippels, J. Betz, A.J. Huis in 't Veld, in Proceedings of the 13th International Symposium on Laser Precision Microfabrication, Washington, 12-15 June 2012

33. E. Ohmura, K. Ogawa, M. Kumagai, M. Nakano, K. Fukumitsu, H. Morita, in Proceedings of the LAMP2009 - the 5th International Congress on Laser Advanced Materials Processing (2009)

34. K. Shimamura, J. Okuma, S. Ohmura, F. Shimojo, J. Phys. Conf. Ser. 402(1), 012044 (2012)

35. G.E. Jellison Jr, D.H. Lowndes, Appl. Phys. Lett. 41(7), 594 (1982)

36. P.C. Verburg, G.R.B.E. Römer, A.J. Huis in 't Veld, Appl. Phys. A: Mater. Sci. Process. 114(4), 1135 (2014)

37. E.V. Zavedeev, V.V. Kononenko, V.M. Gololobov, V.I. Konov, Laser Phys. Lett. 11(3), 036002 (2014)

38. M. Kumagai, T. Sakamoto, E. Ohmura, in International Symposium on Semiconductor Manufacturing (2007)

39. S. Ruffell, J.E. Bradby, J.S. Williams, P. Munroe, J. Appl. Phys. 102(6), 063521 (2007)

40. M.J. Smith, M.J. Sher, B. Franta, Y.T. Lin, E. Mazur, S. Gradečak, J. Appl. Phys. 112(8), 083518 (2012)

41. V. Domnich, Y. Gogotsi, Rev. Adv. Mater. Sci. 3, 1 (2002)

42. A.G. Cullis, N.G. Chew, H.C. Webber, D.J. Smith, J. Cryst. Growth 68(2), 624 (1984)

43. R. Hull (ed.), Properties of Crystalline Silicon (The Institution of Electrical Engineering, 1999)

44. J.T. Wang, C. Chen, H. Mizuseki, Y. Kawazoe, Phys. Rev. Lett. 110, 165503 (2013)

45. C.C. Yang, J.C. Li, Q. Jiang, Solid State Commun. 129(7), 437 (2004) 